\title{
Complex Information Game Problem Based on Artificial Neural Network
}

\author{
Xingfeng Liu,Tiansong Zhou and Zhongxia Zheng \\ 1, 2, 3 School of , Management, Wuhan University of Technology, , P. R. China, 430070 \\ 249763651@qq.coml;
}

\begin{abstract}
The assumption of game theory is that the players in game must be rational. In the game of incomplete information, participants are not completely clear about the game. Therefore, usually there is a probability distribution of strategy selection in game. It is very complicated to know the real game information of the social and economic problems. In fact, the actual situation for many problems is that game players are irrational, or the probability distribution of game players' strategies cannot be gotten, even the strategy sets are not complete (infinite strategy sets).There are many limitations in application of the traditional game theory. In this paper, the concept of complex information game and its Nash equilibrium are presented. It is proved that the complex information game problem can be solved by artificial neural network. An example on how to solve the complex information game problem with artificial neural network is given as well. Researchers hope that more and more scholars can use artificial intelligence theory to analyze the game theory problem. Therefore, the complex information game problems can be dealt more efficiently.
\end{abstract}

Keywords - Artificial Intelligence; Artificial Neural NNetwork; Complex Information; Game Theory

\section{INTRODUCTION}

Game Theory is the most shining new theory in the development of modern economic. Since 1990s, the Nobel economic prize has been awarded to scholars who have an outstanding study on game theory for many times. Therefore, game theory has been put into a new climax.

In 1950, Nash put forward Nash Equilibrium, which laid a theoretical foundation for game problems [1, 2]. After that, many scholars began to study on game theory. Scholars like Selten and Harsanyi carried out dynamic and incomplete analysis based on "Nash Equilibrium", and other ones put forward concepts like "Perfect Nash Equilibrium", "Bayesian Nash Equilibrium" and "Perfect Bayesian Nash Equilibrium". All of these have formed modern game theories [3, 7]. In China, scholars also make comprehensive researches and applications on game theories [8-12].

Neural Network is an important field in which researchers explore to imitate brain information processing system. In 1943, psychologist W. S. Mcculloch and mathematician W.H. Pitts built the M-P neuron model. Up to now, the implementation and application on Neural Network model, learning arithmetic and neural computer have achieved many encouraging fruitful results [13-15]. With the development and perfection of neural network, it will definitely play a more significant role in science and technology. Therefore, a new field is to be searched out in game theories with the application of neural network into game theory in this paper.

\section{THE FOUNDATION AND HYPOTHESIS OF GAME THEORY}

In economic literature, the earliest research on game theories was monopoly pricing and production written by Cournot (1838), Bertrand (1883) and Edgeworth (1925) in their paper. However, these are regarded as a special case, because their studies do not change methods that most economists think of problems [16]. In 1944, John von Neumann and Oskar Morgenetern introduced the idea of universal game theories in their famous book named Game Theories and Economic Behaviors, in which they present that most of the economic problems should be treated as a game for analysis [17]. They introduced the notation of the game's expansion formula and standard formula (or strategic type), defined the minimum maximal solutions and proved the existence of the solution of two zero - sum games in all participants.

Nash (1950) proposed the concept that came to be known as the "Nash equilibrium". He extended the concepts of game theories as a way to analyze the non-zero-sum games. The Nash equilibrium strategy requires each participant to respond to pay off at maximum directly against his predicted opponents' strategies, and the predictions about each participant are correct.

Definition 1: strategy combination $\sigma^{*}$ is a Nash equilibrium, if for all participants " $i$ " there is

ui $\left(\sigma i^{*}, \sigma-i^{*}\right) \geqslant u i\left(\mathrm{si}^{*}, \sigma-\mathrm{i}^{*}\right), \mathrm{si} \in \mathrm{Si}$

Nash equilibrium is a strategic combination, which enables every participant's strategy to be the best response to the other participants. This is a natural extension of equilibrium of a particular model that Cournot and Bertrand studied. What's more, it is the starting point for most economic analysis [18]. However, in some cases, it seems that the Nash equilibrium concept is too harsh [19]. Therefore, it is meaningful to make any predictions on the condition that Nash equilibrium cannot be reached. Then, iterated strict dominance and rationalizability concepts come up [20].

Definition 2: The process of successive elimination of iterated strict dominance strategies is as follows:

Set $\mathrm{SiO} \equiv \mathrm{Si}, \quad \sum \mathrm{i} 0 \equiv \sum \mathrm{i}$. Recursive definition of Sin: $\operatorname{Sin}=\left\{s i \in \operatorname{Sin}-1 \mid\right.$ no $\sigma i \in \sum$ in-1 for all $s-i \in S$-in-1, there is ui( $\sigma \mathrm{i}, \mathrm{s}-\mathrm{i})>\mathrm{ui}(\mathrm{si}, \mathrm{s}-\mathrm{i})\}$, and define: 
$\begin{aligned} \sum i 0=\left\{\sigma i \in \sum i \mid\right. & \sigma i(s i)>0, \quad \text { si } \in \operatorname{Sin}\}, \quad \text { Set } \\ \text { Si } \infty=\bigcap \infty n=0 \text { Sin. } & \end{aligned}$

The concept of rationalizability is introduced by Bernheim and Pearce (1984), and it can be defined according to the following iterative process:

Definition 3: $\tilde{\sum} \mathrm{i} 0 \equiv \sum \mathrm{i}$, for each recursive definition:

$\tilde{\sum} \mathrm{i} 0=\left\{\sigma \mathrm{i} \in \tilde{\sum} \mathrm{in}-1 \mid \exists \sigma-\mathrm{i} \in \times \mathrm{j} \neq \mathrm{i}\left(\right.\right.$ Convex hull of $\tilde{\sum}$ in-1) for all $\sigma \mathrm{i}^{\prime} \in \tilde{\sum}$ in-1there is ui $\left.(\sigma \mathrm{i}, \sigma-\mathrm{i}) \geqslant \mathrm{ui}\left(\sigma \mathrm{i}^{\prime}, \sigma-\mathrm{i}\right)\right\}$

The rationalizability strategies of participant $\mathrm{i}$ are $\mathrm{Ri}=\bigcap \infty \mathrm{n}=0 \tilde{\dot{\sum}}$ in .

Selten (1965) proved that not all the Nash equilibriums are equally reasonable in the game in which participants took the strategies of "contingent action plan". Thus the concept of "subgame perfection" was introduced to exclude the threats which are dependent on such equilibrium [20]. Harsanyi (1967-1968) proposed a method using a standard game technology to model the situation of incomplete information. In the standard technique, the assumption is that all participants are aware of payment function of other people while participants in the incomplete information are not sure about payments of other people. Bayesian Nash equilibrium is analyzed on the basis of many game theories [21]. Harsanyi's thoughts are mainly used in "mechanism design" problem. These applications include nonlinear price discrimination, optimal auctions, display of public product preferences and inefficiencies of negotiating in incomplete information [22-23].

When the game is incompletely informative and dynamic, the concept of Bayesian Nash equilibrium is too weak, because it is the same as the Nash equilibrium in the dynamic game of complete information, allowing existence of empty threat [24]. Therefore, the scholars extended the perfect idea of sub-game to solve the game of incomplete information [25]. According to restrictions from ascending order in turn, these ideas of solving are ranked from perfect Bayesian equilibrium, Kreps and Wilson (1982) Sequential equilibrium, to Selten (1975) Trembling-hand perfect equilibrium. Scholars such as Xiao Yuanzhang, Park Jaeok, and Vander Schaar Mihaela illustrated these ideas through applications in predatory games and games of passing signals in labor market [26].

In a nutshell, the game theories mainly research on mutual interaction in the formulated incentive structure. Game theories are mathematical theories and methods in the study of fight or phenomena of competitive nature [27]. Game theories consider individual predicted behaviors, actual behaviors, and study their optimization strategies. Currently, game theories have become a standard tool for economic analysis. In the meanwhile, game theories have been put into a wide range of applications in economics, biology, international relation, computer science, political science, military strategy, and other disciplines as well.

\section{THE PROBLEMS AND SHORTCOMINGS OF MODERN GAME THEROY}

The game problem, which is the problem that some individuals, teams or organizations, in the face of certain environmental conditions, under certain rule, simultaneously or successively, one or more times, choose from their behaviors or strategies, which allow selection of the process and to be implemented, and obtain the corresponding results.

From the game theory definition, researchers can find that a typical game should have four aspects:

(1) The participants of game (game player).

(2) Strategy set the game.

(3) The order of game.

(4) The benefit of game.

In order to seek the solution (equilibrium) of game, there is a key assumption that game participants are completely rational. However, the actual situation is not always so. In many cases, the game is the rational part (semi- rational or irrational). Take a look at the following example.

\begin{tabular}{|c|c|c|}
\hline \multirow{3}{*}{ Attack } & \multicolumn{2}{|c|}{ THE GOVERNMENT } \\
\hline & DEFENSE & NO DEFENSE \\
\hline & $-100,-20$ & $-100,-1000$ \\
\hline & $0,-10$ & 0,0 \\
\hline Figure 1. & \multicolumn{2}{|c|}{$\begin{array}{l}\text { Game between terrorists } \\
\text { and the government }\end{array}$} \\
\hline
\end{tabular}

In game problem shown such as the example in Figure 1 , the strategy set of Terrorists are \{Attack, No attack\}. The strategy set of government are \{Defense, No defense $\}$. Since the terrorists" benefit to attack is " -100 " and no attack is " 0 ", so a rational player will not choose to attack. The Nash equilibrium is (No attack, No defense), both sides benefit from $(0,0)$, However, that is not the case, because the game players (terrorists) are irrational. If the government selects strategies in accordance with "No defense", it may suffer a great deal. Both sides in the game in real life are the results of (the attack, defense), here it is regarded as "semi-rational equilibrium". From the game problem above, it can be found that the judgment of the game players' type is very important. The results whether game players are rational will not directly affect the process and results of the game. Therefore, the hypothesis of game theory is that game players are rational. So, what is the method and basis for the judgment if the game players are irrational?

Researchers believe that these problems of the Game theory can be solved by artificial neural network. In this paper, the type of game participants can be judged by competitive learning network on the game problem above. Now researchers prepare a basic competitive learning network. The network consists of two layers, the first layer is the input layer, composed of a processing unit to receive the input mode; the second layer is the competitive layer, competition unit to respond to input patterns, namely, the said category input mode. The input layer unit connection to the competition unit for connection modes of the connection weight is adjustable. See Fig .3. 


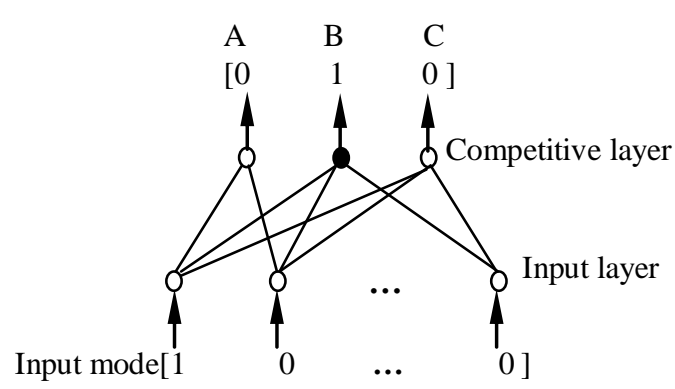

Figure 2. Competition network with two layers

Figure 4 is a processing unit competitive layer. The assumption is that each competition unit (each type of Game Party) and the right value is always 1 , i.e. $\sum \mathrm{Wjt}=1$. When the initial weights of network learning take a random number of a group of small, the input mode is $0 / 1$ vector binary. For the jth competition unit, the sum of input is $\sum \mathrm{Wjt}=1$. With the maximum input sum competition layer unit is determined to be the winner, whose output is 1 and other cell state is 0 , i.e.

$$
x_{j}^{c}= \begin{cases}1 & S_{j}>\max \left(S_{k}, k \neq j, k=1,2, \cdots n\right) \\ 0 & \text { Others }\end{cases}
$$

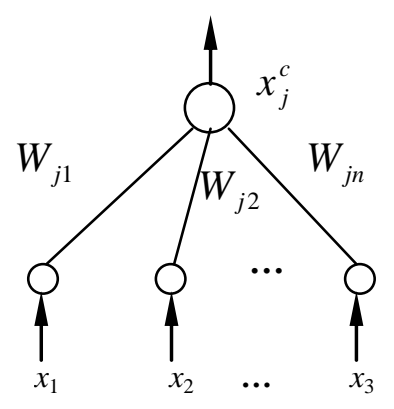

Figure 3. processing unit of competitive layer

In formula (2), xcj represents the jth unit's output state of competition layer. For an input pattern, weights are updated when the competition winner unit is determined by the rule $\Delta \mathrm{Wjt}=\eta(\mathrm{xi} / \mathrm{m}-\mathrm{Wjt})$, wherein said, $\eta$ is learning factor, $0 \leqslant \eta \leqslant 1 ; m$ represents the input layer number of unit 1 .

On top of the game, by collecting some information researchers can determine the corresponding input mode. After the judgment of neural network on a side of the game, the other game parties can choose their own strategies, so as to achieve the "semi rational equilibrium" or "rational equilibrium" (Nash equilibrium).

In addition, researchers consider that the self-organizing map neural network (SOM), the counter propagation neural network $(\mathrm{CP})$, the adaptive resonance theory neural network (ART) and the competitive learning network all can be applied here.

\section{COMPLEX INFORMATION GAME PROBLEMS}

In the modern game theory, game is composed of three elements: participants' collection $i \in I$, strategic space of $\mathrm{Si}$ in every participant $\mathrm{i}$ and revenue function ui [28]。

In order to solve the game (i.e.: Nash Equilibrium), one of the key assumption is all the game parties are rational [29]. That is to say, game analysis and strategic participants make decision completely based on the revenue function. To minimize his profit function is the only pursuit [30].

At present, in most cases, pay off functions and utility level are adopted to represent the profit function. In the real situation, it is difficult to confirm the profit function for participants. Especially in adversarial game problems, apart from minimizing players' profits, it is more important for them to beat the opponents [31]. A typical example of game problem is whether terrorists use "suicide bombers" to attack and whether the government takes measures to prevent. In some sense, game players have preferences [32]. In terms of this issue, FengJun Wen has made a research on it by the use of ordinal information game theory [33].

Regarding to a multi-stage dynamic game, there are some more complicated situations: some players do not know the profits of other game players (i.e. incomplete information game in the modern game theory). Meanwhile, some players can not completely know what strategies other players may take [34]. (In other words, some players can only predict some possible strategies other players may take and never know all the strategies). At present, game problems of this kind are more complicated; therefore, nobody defines or studies them [35]. And because of this, this paper is to put forward the concept of complex information game in terms of these problems.

Definition 4: For the problem of a multi-stage dynamic game, if one player or mutual players do not know profits each other and do not know all strategies of other players or just know some strategies of other players, game problems of this kind are called complex information game.

For instance, researchers consider the confrontation between terrorists and government. The government knows some of terrorists' strategies such as bombing the embassy, kidnapping government officials but doesn't know all the strategies of the terrorists. When the terrorists have adopted new strategies such as "9.11" attack, the government begin to know terrorists' strategies, but still cannot know all strategies of terrorists, because terrorists take new strategies in new games. Also, terrorists only know current precautions the government has taken and do not know the government's new strategies.

\section{NASH EQUILIBRIUM OF COMPLEX INFORMATION GAME}

According to the definition of complex information game, complex game problems should be a multi-stage game process in which every stage of the Nash equilibrium is not easy to determine. At the same time, it has certain randomness. And they cause huge obstacles to solve problems of this kind. Therefore, researchers must solve the problems by escaping from the traditional game of Nash equilibrium. With the development of the research on artificial intelligence, researchers think the neural network can provide a solution for the complicated information game. In fact, as an important field of artificial intelligence, neural network has been put into applications in many game problems, such as Chinese checker, go, tic-tac-toe, chess, and poker game. There are 
also many successful models. In a chess game problem, though two people in the game are generally believed to get involved in a game problem, game problems of this kind of are not recognized or classified. According to definition 1, obviously, the game of chess problem belongs to the complex information game.

Definition 5: As for the problem of a complex information game, at every stage, both players believe their own game strategy is optimal strategy under the current state of information. This strategy is called the Nash equilibrium of complex information game.

At each stage, when the Nash equilibrium is reached, it does not mean that players in each side are in a state of steadiness. In fact, with the development of the play and increase of the strategies, "optimal" strategies in earlier stages may be not the most optimal in later stages, but this does not affect the formation of Nash equilibrium. This is due to the complex dynamic information, at the same time, it is featured with time irreversibility, and namely, strategies chosen only relate to the current information and state. It has nothing to do with the previous balance, so Nash equilibrium in each stage has certain randomness, and this is quite different with the traditional game.

\section{AN EXAMPLE ABOUT THE APPLICATION OF ARTIFICIAL NEURAL NETWORK IN COMPLEX INFORMATION GAME}

Just now, researchers discussed the feasibility of the use of neural network to solve complex information game problem. Researchers will take an example to discuss how to use neural network to solve complex information game problem.

Although in Bayesian game it can be known that a little about the "types" of other parties, in general, a game party at least knows the size in all sorts of "types" in the other players, namely the "judgment" of the probability distribution of all kinds of "types". Therefore, "reverse induction method" is used to solve the "Bayesian Nash equilibrium".

One of the important aspects in the above game theory is the benefits of the game parties. Actually benefits of all parties are due to incomplete information in real life. So-called problems of incomplete information game are also called the "Bayesian game". They refer to some players' benefits or profit function which at least one game party is not completely clear.

If each game party can not make judgments on the benefits of the game parties or their judgments do not conform to the actual situations, then what should researchers do? Take an example of game problems of the melon seller and buyer (Figure 4). When the seller sells the water melons with high price, there are two kinds of benefits: - 1 and 1, then there are two different Nash equilibrium of the game, namely, (low price, buy) and (high price, buy).Therefore, if the melon seller doesn't know the benefits of the buyer when he sells melons, the seller will not be able to make a choice.

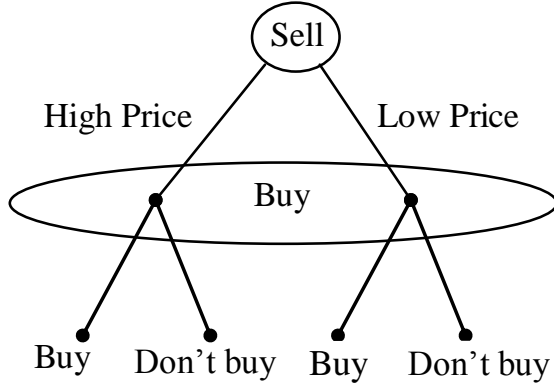

$$
\begin{aligned}
& (-1,3)(0,0)(2,1)(0,0) \\
& (1,3)(0,0)(2,1)(0,0)
\end{aligned}
$$

Figure 4. Game between melon sellers and buyers

As a matter of fact, the game is still in progress. That is to say, choices that high price or low price melon sellers make are not in accordance with the buyers' benefits. (because he couldn't know the benefit judgment of the different buyers or melon buyers' benefits differ from each other), now melon sellers make strategic choice which is subject to certain information they have already known, then melon buyers decide to buy or not to buy according to sellers' offer. Right now, the game is actually an "order" game, namely, the game results are not made by the "reverse induction", but rather by game parties who choose their strategies in a certain order finally. Now researchers use $\mathrm{M}-\mathrm{P}$ model neurons (threshold neurons) to simulate the game process, as is shown in Fig.5.

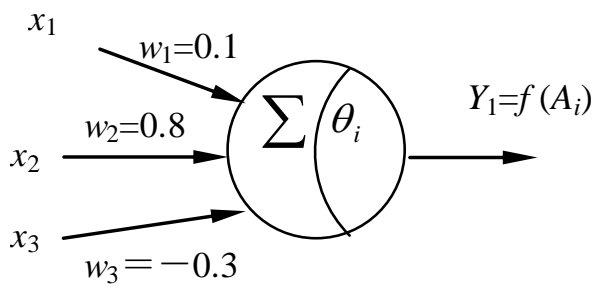

Figure 5. $\quad \mathrm{M}$ - $\mathrm{P}$ model neurons

$\mathrm{x} 1, \mathrm{x} 2, \mathrm{x} 3$ are assumed to be three neuron inputs, they represent three factors that melon sellers decide to choose high or low price; wj represents the connection strength between neuronal synaptic and the $\mathrm{j}$ factor. Ai represents the total output of the neuron; Y1 shows the output of the neuron; $\theta \mathrm{i}$ shows neuron threshold value, then the output of the neutron can be expressed as

$$
y_{i}=f\left(A_{i}\right)= \begin{cases}1 & A_{i}>0 \\ 0 & A_{i} \leq 0\end{cases}
$$

there into $\quad A_{i}=\sum_{j=1}^{n} w_{j} x_{j}-\theta_{i}$

The melon sellers' reactions on the various situations are assumed as follows:

$$
\begin{array}{lllllllll}
\mathrm{x} 1: & 0 & 0 & 0 & 0 & 1 & 1 & 1 & 1 \\
\mathrm{x} 2: & 0 & 0 & 1 & 1 & 0 & 0 & 1 & 1 \\
\mathrm{x} 3: & 0 & 1 & 0 & 1 & 0 & 1 & 0 & 1 \\
\mathrm{Y} 1: & 0 & 0 & 1 & 0 & 0 & 0 & 1 & 0
\end{array}
$$

$\mathrm{x} 1, \mathrm{x} 2, \mathrm{x} 3$ represent the melons are good or bad (1/0), the size of the market demand (1/0), and melon sellers are eager to sell or not (1/0). Y1 shows that melon sellers 
choose high price or low price to sell (1/0). Therefore, weight and threshold of neurons must meet the following inequality group.

$$
\left\{\begin{array}{l}
w_{1}+w_{2}+w_{3}-\theta<0 \\
w_{1}+w_{2}-\theta>0 \\
w_{1}+w_{3}-\theta<0 \\
w_{2}+w_{3}-\theta<0 \\
w_{1}-\theta<0 \\
w_{2}-\theta>0 \\
w_{3}-\theta<0 \\
-\theta<0
\end{array}\right.
$$

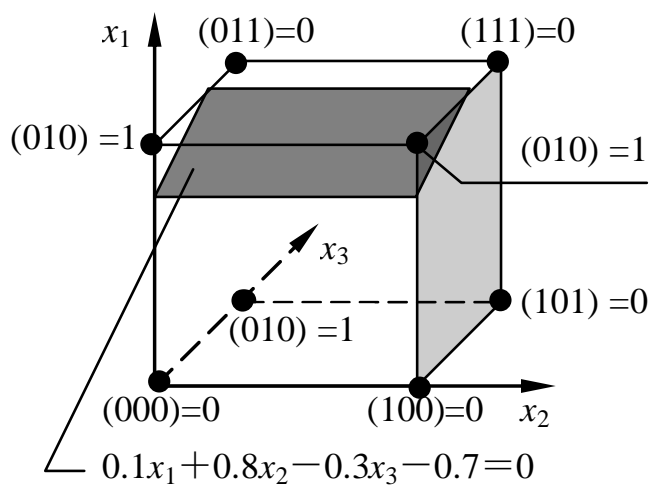

Figure 6. M-P geometric representation of the model solution

As is shown in Fig .6, $0.1 \times 1+0.8 \times 2-0.3 \times 3-0.7=0$ is a feasible decision plane. Therefore, decision model of the neuron of melon seller is assumed as follows: $w 1=0.1$, $\mathrm{w} 2=0.1$, w3 $=0.3, \theta=0.7$ Also, the melon buyers' reactions on the high or low offer of the melon seller (1/0) and the quality of the melon (1/0) are assumed as follows:

Y1: $\quad 0 \quad 0 \quad 0 \quad 1 \quad 1$

Y2: $\quad 0 \quad 1 \quad 001$

Fi: $\quad 1 \quad 1 \quad 0 \quad 1$

$\mathrm{Fi}$ is the decision of buying or not buying (1/0)of the “i”th melon buyer. Although there are many melon buyers, their Fi output model is different. Anyway, researchers can make the output of the above model as most people's output. Therefore, the neuron weight and threshold value of $\mathrm{M}$ - P model of melon buyer meets the following inequality group:

$$
\left\{\begin{array}{l}
W_{1}+W_{2}-\theta>0 \\
W_{1}-\theta<0 \\
W_{2}-\theta>0 \\
-\theta>0
\end{array}\right.
$$

Also, it is known that " $-0.5 \mathrm{Y} 1+0.5 \mathrm{Y} 2+0.3=0$ " is a feasible decision-making straight line. Therefore, "W1 = $0.5, \mathrm{~W} 2=0.5, \theta=-0.3$ " is assumed to be neuron decision model of the melon buyers. The game result is as follows:

$\begin{array}{lllllllll}\text { (1) (2) } & \text { (3) } & \text { (4) } & \text { (5) } & \text { (6) } & \text { (7) } & \text { (8) } \\ \text { X1: } 0 & 0 & 0 & 0 & 1 & 1 & 1 & 1 \\ \text { X2: } 0 & 0 & 1 & 1 & 0 & 0 & 1 & 1 \\ \text { X3: } 0 & 1 & 0 & 1 & 0 & 1 & 0 & 1 \\ \text { Y1: } 0 & 0 & 1 & 0 & 0 & 0 & 1 & 0 \\ \text { Fi: } 1 & 1 & 0 & 1 & 1 & 1 & 1 & 1\end{array}$

From the result, researchers know that there is only one situation that most melon buyers do not buy melons, and researchers have the following conclusion:

(1) In the two cases of (1) and (2), the melons are not good. Few people buy the melons (melon buyers in this article refer to people who have intention to buy melons, i.e. people who are willing to ask the price. Many people who do not want to buy melons will not ask the price and buy melons. They do not take part in the game.), so the market is very small. Whatever melon sellers are anxious to sell the melons, they will only sell melons at a low price (he cannot lose money in business), melons buyers will still buy the melons. This is a failure of the market.

(2) In case (3), melons are not good, but they are few(out of stock), so the market is comparatively bigger, therefore melon sellers are not anxious to sell in a hurry, the market price is very high, melon buyers(most of melon buyers) do not buy melons. This is a complete failure of the market (stock market).

(3) In case (4), the melons are not good, but a lot of people buy them. Melon sellers are eager to sell melons and choose the low price (for a high-volume, low-margin business), then people buy melons. This is a more successful market.

(4) In the cases of (5), although melons are good, only a few people buy melons) (for instance, the autumn arrives or the weather is too cold), no matter melon sellers are keen to sell melons in a hurry or not, they sell melons at a low price, then people buy melons. This is a more successful market.

(5) In case (7), melons are good, there are also many melon buyers, melons are sold at a high price, many people buy melons. This is a more successful market.

(6) In case 8, melons are good, there are many melons buyers, melons are sold at low price, people buy melons. This is a very successful market.

To solve the problem, of course, the final stopping point should go back to the neural network design problem. In this article, researchers design suitable network based on the current information and use the past information to have network training by which a game is simulated. And, of course, this is just viewpoints in this article because good effects still need further researches.

\section{CONCLUSIONS}

A brand-new concept of complex information game and its Nash equilibrium are proposed in this paper. At the same time, in view of deficiencies in game theory, researchers try to use neural network to solve these problems, and make corresponding conclusions. From our demonstrations, researchers find that it is feasible to apply the neural network to the game theories and researchers hope that there will be more and more developments and applications of the neural network theories into the game theories, thus a new field is explored in the game theories. 


\section{REFERENCE}

[1] Nash. J. The bargaining problem [J]. Econometrica, 1950, 18:155-162

[2] Nash. J. Equilibrium points in n-person games [J]. Proceedings of the National Academy of Sciences, 1950, 36:48-49

[3] Selten. R. Re-examination of the perfectness concept for equilibrium points in extensive games [J]. International ournal of Game Theory, 1965, 4:25-55

[4] Harsanyi. J. Games with incomplete information played by Bayesian players [J]. Management Science, 1967-68, 14:159-182, 320-334, 486-502

[5] Reinganum J F. Dynamic games of innovation [J]. Journal of Economic Theory, 1981, 25(1):21-41.

[6] JorgenW W. Evolution, rationality and equilibrium in games [J]. European Economic Review, 1998, 42(3/5): 641-649.

[7] Smit H T J, Ankum L A. A real options and game-theoretic approach to corprate investment strategy under competition [J]. Financial Management. 1993, 22(3):241-250.

[8] Liu Weibing, Wang Xianjia. Study on evolutionary games based on PSO-neural networks $[\mathrm{J}]$. Systems Engineering and Electronics, 2007, 29( 8) : 1283 - 1285. (in Chinese)

[9] Zheng Junjun, Han Xiao, Zou Zuxu, et al. Analysis on venture capitalists'strategies in IPO market based on evolutionary game [J]. Journal of Management Sciences in China, 2012, 15( 2) , 72 - 82. (in Chinese)

[10] JiangGuo-yin, $\mathrm{Hu}$ Bin, WangHuan-huan. Agent-based simulation research on collaboration strategy fordynamic coalition of service providers based on the evolution game [J]. Chinese Journal of Management Science, 2009, 17(2): 86-92. ( in Chinese)

[11] Zhu Qinghua, Dou Yijie. An evolutionary model between governments and core-enterprises in green supply chains $[\mathrm{J}]$. Systems Engineering: Theory \& Practice, 2007, 12( 12) : 85-89. (in Chinese)

[12] Li Yanbo, Liu Songxian. A game analysis between director departments of government and food corporations in information asymmetry $[\mathrm{J}]$. Chinese Journal of Management Science, 2006, 14: 197-200. (in Chinese)

[13] Yang G D, Pan D F, Fan Y F. A method for the environmental quality assessment of surface water based on artificial neural networks [J]. Journal of Systems Science and Systems Engineering, 2000, 9(1):72-76.

[14] Eisenbeis R A.Pitfalls in the application of neural networks: the case of bank failure Predictions [J]. Management Sciences, 1992, 19(4):926-947

[15] Tae Yoon Kim, Kyong Joo Oh, I nsuk Sohn, ChanghaHwang. Usefulness ofArtificial Neural Networks for Early Warning System of Economic Crisis [J]. Expert Systems with Applications, 2004,: 11(5): 43-45

[16] Diffo Lambo, L, Tchantcho, B,Moulen, J. Comparing influence theories in voting games under locally generated measures of dissatisfaction. [J].International Journal of Game Theory. 2012, 41(3): 719-731.

[17] Masek, Martin,Murcia, Karen; Morrison, Jason. Learning in Transformational Computer Games: Exploring Design Principles for a Nanotechnology Game. [C]. World Education Research Association (WERA) Focal Meeting, 2012, 510-542
[18] Krawec, Walter. Analyzing n-player impartial games. [J] International Journal of Game Theory. 2012, 41(2): 345-367.

[19] Navidi Hamidreza, Bashiri Mahdi, Messi Bidgoli Masume. A heuristic approach on the facility layout problem based on game theory. [J]. International Journal of Production Research. 2012 50(6): 1512-1527.

[20] Grün Christine. A Probabilistic-Numerical Approximation for an Obstacle Problem Arising in Game Theory. [J]. Applied Mathematics \& Optimization. 2012, 66(3): 363-385.

[21] Kawasaki Hidefuui, Kira Akifumi, Kira Shinpei. An Application of a discrete fixed point theorem to a game in expansive form [J]. Asia-Pacific Journal of Operational Research. 2013,30(3):1-7.

[22] Bolton Gary,Brosig-Koch Jeannette. How do coalitions get built? Evidence from an extensive form coalition game with and without communication. [J]. International Journal of Game Theory. 2012, 41(3): 623-649.

[23] [23] Li Lei, Li Xueliang. The covering values for acyclic digraph games. [J]. International Journal of Game Theory. 2011,40(4) 697-718.

[24] Ye Ye,Wang Lu,Xie Nenggang. Parrondo's Games Based on Complex Networks and the Paradoxical Effect. [J]. PLoS ONE. 2013, 8(7): 1-11

[25] Wang Xu, Kwong Sam,Zhang Yun. Applying Game Theory to Rate Control Optimization for Hierarchical B-Pictures. [J] Broadcasting. 2013, 59(4): 591-601.

[26] Mojica-Nava Eduardo, Macana Carlos Andres, Quijano Nicanor. Dynamic population games for optimal dispatch on hierarchical microgrid control. [J]. Systems, Man \& Cybernetics Systems. 2014, 44(3): 306-317.

[27] Vitorino Maria Ana. Empirical Entry Games with Complementarities: An Application to the Shopping Center Industry. [J]. Journal of Marketing Research. 2012, 49(2): 175-191.

[28] Grenadier S R. Game Choices: The intersection of real options and game theory [M]. London: Risk Books, 2000.

[29] Huang Z, Li S X. Co-op Advertising Models in Manufacturer Retailer Supply Chains: A Game Theory Approach [J]. European Journal of Operational Research, 2001, 135 ( 3) : 527-544.

[30] Leng M, Zhu A. Side-Payment Contracts in Two-Person Nonzero Sum Supply Chain Games: Review, Discussion and Applications [J]. European Journal of Operational Research, 2009, 196( 2) : 600-618.

[31] Prasad A, Sethi S P . Competitive Advertising under Uncertainty: A Stochastic Differential Game Approach [J]. Journal of Optimization Theory and Applications, 2004, 123 ( 1 ) : 163-185.

[32] Cellini R, Lambertini L. Advertising in a differential Oligopoly Gam [J]. Journal of Optimization Theory and Applications, 2003, 116( 1) : 61-81.

[33] FengJun Wen. Game theory with ordinal data inputs [J]. Chinese Journal of Management Science, 2002,5(4):83-87 ( in Chinese)

[34] Navas J, Marín-Solano J. Interactions between Government and Firms: a Differential Game Approach [J]. Annals of Operations Research, 2008, 158: 47-61.

[35] DuW B, CaoX B, HuM B, et a.l Effects of expectation and noise on evolutionary games $[\mathrm{J}]$. Physica A, 2009, 388 (11): 2215-2220. 